\title{
DOUBLE CONTINUUM MODEL OF EVOLUTION OF PRECURSOR DROPLETS IN THE FLOW OF HEAT TRANSFER MEDIUM OF A PLASMA CHEMICAL REACTOR
}

\author{
Ilya S. Zhukov ${ }^{1}$, Sergey S. Bondarchuk ${ }^{1}$, and Boris V. Borisov ${ }^{2, *}$ \\ ${ }^{1}$ Institute for Problems of Chemical \& Energetic Technologies of the Siberian Branch of the Russian \\ Academy of Sciences, 659322 Byisk, Russia \\ ${ }^{2}$ National Research Tomsk Polytechnic University, 634050 Tomsk, Russia
}

\begin{abstract}
A double continuum physical and mathematical model of evolution of precursor in a vaporizing droplet of low-concentration solution of metal salt in the process of its heating in the flow of heat transfer medium of a plasma chemical reactor is represented in this paper. This model was developed to study the morphology of ceramic powder particles depending on solution characteristics and parameters of a hightemperature gaseous heat transfer medium which determine heat and mass transfer with a droplet medium.
\end{abstract}

Plasma chemical synthesis including thermo-chemical decomposition of liquid sprayed agents(precursors) in the flow of a high-temperature gaseous heat transfer medium [1,2] is one of the most promising technologies of production of ceramic powders with controlled parameters. The term "spray pyrolysis" is used for this technology in English [3].

Analysis of separate stages of plasma chemical synthesis nave indicated [1-4] that powder particle morphology is formed at the stage of droplet evaporation. Simultaneous solvent evaporation from the droplet surface and salt diffusion towards the center of the droplet is the basic process determining product structure. The relation between the rates of these processes not only determines the size of a salt "residue" particle formed in the process of solvent evaporation but also its structure - nut-type (with a relatively empty nucleus) or solid (without any cavities).

Physical and mathematical models of processes taking place in a plasma chemical reactor are necessary to provide controlled operation of the latter in order to produce particles with specified characteristics. At the same time, a number of well-known works are based on very significant simplifying assumptions in description of processes in the droplet of solution [1-4], particularly on approximate equality of solvent density and admixture density. This fact significantly reduces predictability of characteristics of synthesized metal oxide powders and their morphology (internal structure). The offered double continuum model of evolution of precursor droplet considers diffusion of an admixture with the density different from the density of the solvent.

*Corresponding author: bvborisov@tpu.ru 
The basic assumption is the condition of conservation of volume of each component of the mixture in the process of its movement determined by the increase of mass fraction of salt at the boundary of precursor droplet due to solvent evaporation. This assumption leads to the following

$$
\frac{\varrho^{s}}{\varrho^{s o}}+\frac{\varrho^{m}}{\varrho^{m o}}=1,
$$

where $\varrho^{s}, \varrho^{m}$ - reduced densities (in the mixture) of salt and solvent;

From mass conservation equations for components

$$
\frac{\partial \varrho^{s}}{\partial t}+\frac{\partial}{\partial r}\left(r^{2} \varrho^{s} u^{s}\right)=0, \frac{\partial \varrho^{m}}{\partial t}+\frac{\partial}{\partial r}\left(r^{2} \varrho^{m} u^{m}\right)=0
$$

taking into account relation (1) we can obtain

$$
\frac{\partial}{\partial r}\left(\varrho^{s} u^{s}+\varrho^{m} u^{m}\right)=0
$$

where $t$ - time, $r$-coordinate oriented from the centre of the droplet,

$u^{s}, u^{m}$ - velocities of mixture components.

Let us assume that salt velocity for the whole process is determined by the Fick's law using diffusion coefficient $D$ and gradient of salt concentration $c$

$$
u^{s}=D \frac{\partial c}{\partial r} .
$$

Mass fraction $\mathrm{c}$ is linked with reduced densities of the mixture by the following relation

$$
c=\varrho^{s} /\left(\varrho^{s}+\varrho^{m}\right) \text {. }
$$

Taking into account relation (2) for an elementary volume $d V$, bounded by surface $S$, laws of conservation of mass for mixture components can be represented as follows

$$
\begin{aligned}
& \frac{\partial}{\partial t} \int_{V} \varrho^{s} d V+\int_{S} \varrho^{s} u^{s} d S+\int_{S} \varrho^{s} v d S=0, \\
& \frac{\partial}{\partial t} \int_{V} \varrho^{m} d V+\int_{S} \varrho^{m} u^{m} d S+\int_{S} \varrho^{m} v d S=0, \\
& u^{s}=D \frac{\partial c}{\partial x}, \varrho^{m} u^{m}=\frac{\varrho^{m o}}{\varrho^{s o}} \varrho^{s} u^{s} .
\end{aligned}
$$

Convective mass transfer for a surface $S$ moving with a velocity $v^{*}$ is determined by droplet size reduction due to solvent evaporation.

Boundary conditions are defined on the basis of the physics of the process by the following relations.

$$
\left.u^{s}\right|_{r=0}=\left.u^{s}\right|_{r=R}=0 ;\left.\quad u^{m}\right|_{r=0}=\left.u^{m}\right|_{r=R}=0 .
$$

At the main stage of the process when solvent evaporates under the influence of external heat flow it can be assumed that droplet surface temperature is equal to boiling temperature $T_{\text {vap }}$ for given ambient pressure.

In the framework of the model of centrally symmetric reduced film $[4,5]$ (the radius of this film is determined by the condition of quasi-stationary heat exchange with the medium 
taking into account heat consumption for evaporation and vapor superheating up to the temperature of a heat transfer medium $T_{e}$ ) the relation for current rate of droplet radius reduction $v^{*}$ can be written as follows

$$
v^{*}=-\frac{d R}{d t}=\frac{\lambda_{g}}{\varrho^{m} R c_{\text {vap }}} \cdot \mathrm{Nu} \cdot \ln (1+\mathrm{B}), \quad u^{s}=D \frac{\partial c}{\partial r} .
$$

where $B=c_{\text {vap }}\left(T_{e}-T_{\text {vap }}\right) / H_{\text {vap }}-$ Spalding number; $\mathrm{Nu}=2+0.16 \sqrt[3]{\mathrm{Re}_{\Delta}^{2}}-$ Nusselt number;

$\mathrm{Re}_{\Delta}$ - Reynolds number of relative particle movement in a gas flow;

$\lambda_{\mathrm{g}}$ - gas heat transfer coefficient; $c_{\text {vap }}-$ specific heat capacity of vapors; $H_{v a p}$ - heat of evaporation.

The following difference scheme can be used to solve these equations.

$$
\begin{gathered}
\frac{\bar{\varrho}_{i}^{s} \bar{V}_{i}-\varrho_{i}^{s} V_{i}}{\Delta t}=r_{i}^{2} \varrho_{i-\frac{1}{2}}^{s} u_{i-\frac{1}{2}}^{s}-\left(r_{i}+\Delta r\right)^{2} \varrho_{i+\frac{1}{2}}^{s} u_{i+\frac{1}{2}}^{s}+r_{i}^{2} \varrho_{i-1}^{s} v_{i-\frac{1}{2}}-\left(r_{i}+\Delta r\right)^{2} \varrho_{i}^{s} v_{i+\frac{1}{2}} ; \\
\frac{\bar{\varrho}_{i}^{m} \bar{V}_{i}-\varrho_{i}^{m} V_{i}}{\Delta t}=r_{i}^{2} \varrho_{i-\frac{1}{2}}^{m} u_{i-\frac{1}{2}}^{m}-\left(r_{i}+\Delta r\right)^{2} \varrho_{i+\frac{1}{2}}^{m} u_{i+\frac{1}{2}}^{m}+r_{i}^{2} \varrho_{i-1}^{m} v_{i-\frac{1}{2}}-\left(r_{i}+\Delta r\right)^{2} \varrho_{i}^{m} v_{i+\frac{1}{2}} ; \\
r_{i}=i \cdot r, V_{i}=\left(r_{i}^{3}-r_{i-1}^{3}\right) / 3, i=0,1, \ldots, N ; \Delta r=R / N ; \\
u_{\frac{1}{2}}^{s}=0, u_{i-\frac{1}{2}}^{s}=D \frac{c_{i}-c_{i-1}}{\Delta r}, i=2,3, \ldots, N ; \\
u_{i+\frac{1}{2}}^{s}=D \frac{c_{i+1}-c_{i}}{\Delta r} ; i=1,2, \ldots, N-1 ; u_{N+\frac{1}{2}}^{s}=0 \\
\varrho_{i \pm \frac{1}{2}}^{m} u_{i \pm \frac{1}{2}}^{m}=\frac{\varrho^{m o}}{\varrho^{s o}} \varrho_{i \pm \frac{1}{2}}^{s} u_{i \pm \frac{1}{2}}^{s}, i=1,2, \ldots, N .
\end{gathered}
$$

Overline over variables indicates their value at the next time layer. A stencil of the difference scheme is given in fig. 1 , where $f=\left(\rho^{s} ; \rho^{m} ; v ; u^{s} ; u^{m}\right)$.

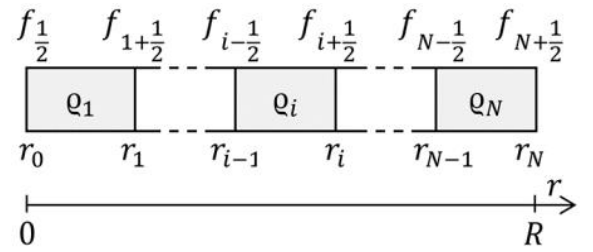

Fig. 1. Stencil of the difference scheme.

Thus, a double continuum model of precursor evolution for diffusion of admixture in the process of movement of vaporizing droplet medium along the loop of a plasma chemical reactor is formulated in this paper. A mass-conservative difference scheme is offered for numerical implementation of the model.

\section{Acknowledgments}

The reported study was funded by RFBR, according to the research project No. 16-3860031 mol_a_dk. 


\section{References}

1. A.L. Suris, Plasma chemical processes and apparatus (Khimiya, Moscow, 1989) [in Russian]

2. V.K. Larin, V.M. Kondakov, E.N. Malyi et al., Izv. vuzov. Non-ferrous metallurgy 5 (2003) [in Russian]

3. G.L. Messing, S.-C. Zhang, G.V. Jayanthi, J. American Ceramic Society 76, 11 (1993)

4. I.S. Bondarchuk, I.A. Zhukov, B.V. Borisov, MATEC Web Conf. 23 (2015)

5. V.V. Pomerantsev, K.M. Arefyev, D.B. Akhmedov et al., Fundamentals of practical combustion theory (Energy, Moscow, 1986) [in Russian] 